\title{
Actualización bibliográfica sobre el uso de preparaciones ricas en plaquetas en la cicatrización de heridas
}

\section{Updated review of the use of platelet rich preparations in wound healing}

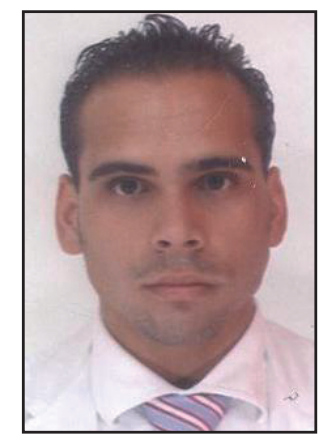

Dr. David Mateo de Acosta

\author{
Mateo de Acosta Andino, DA. ${ }^{*}$ Porres Aguilar, M. ${ }^{\star \star}$, Vázquez Saldaña, DG. ${ }^{* \star *}$, Makipour, Jr. J..***, Bedolla E. ${ }^{* \star \star *}$
}

\section{Resumen}

Las plaquetas poseen más de 20 factores de crecimiento en sus gránulos. Su secreción promueve la reparación de heridas. La cicatrización es un intrincado proceso en el cual intervienen no solo los factores plaquetarios proangiogénicos sino también factores antiangiogénicos. La preservación por congelación de las plaquetas empleada para su preservación, desestabiliza y activa la membrana plaquetaria alterando sus propiedades. En el campo del tratamiento de heridas recalcitrantes en pacientes debilitados o con enfermedades crónicas, el uso de preparaciones plaquetarias en forma de plasma fresco congelado, las preparaciones plaquetarias sonicadas, las preparaciones congeladas y deshidratadas y el uso de fragmentos de dermis alogénica, confiere efectos beneficiosos, logrando mejores tiempos de cierre en las heridas y menor alteración de la morfología de la cicatriz final.

En el presente artículo, describiremos los estudios que han demostrado estos efectos, así como las encrucijadas actuales y los estudios que debemos esperar en un futuro no lejano.
Abstract

Platelets store more than 20 growth factors in their granules. Their secretion promotes wound healing and repair. The intricate wound healing process involves not only the angiogenic platelet factors but also de anti-angiogenic ones. The process of platelet preservation, such as freezing, disrupt the platelet membrane structure and their angiogenic properties causing platelet activation. In the field of wound healing and the management of recalcitrant wounds in debilitated and chronically ill patients, there have been several studies. These studies have focused on the use of the different platelet preparations such as fresh frozen plasma, frozen-dried platelet rich plasma, sonicated platelet rich plasma and the use of alogenic acelular dermis. Platelet preparations posses positive effects on wound healing with little alterations of the final scar tissue.

In this article we will describe and discuss the studies that have involved the use of these platelet rich preparations in the filed of wound healing and we will also state the road blocks and the studies that will come in the near future regarding the use of platelets products in the field wound healing.
Palabras clave Cicatrización de heridas, Plasma

Rico en Plaquetas.

\section{Código numérico}

\footnotetext{
* Cirugía General, Departamento de Cirugía, Universidad de Illinois (UIC - College of Medicine), Chicago, Illinois, USA.

** División de Cuidados Intensivos Pulmonares, Universidad de Utah (Health Sciences Center - School of Medicine), Salt Lake City, Utah, USA

*** Cirugía General, Plástica y Reconstructiva, Departamento de Cirugía, Hospital General Juárez de México (SSA), México DF, México.

**** Cirugia General, Departamento de Cirugía General, Universidad de Oklahoma (OU - Health Sciences Center), Oklahoma City, Oklahoma, USA.
} 


\section{Introducción}

La agregación plaquetaria, que es el proceso inicial de la hemostasia, estimula la liberación de factores de crecimiento que regulan la reparación de heridas (Tabla I), entre los que se encuentran el factor de crecimiento derivado de plaquetas - BB (PDGF-BB, Platelet Derived Growth Factor), el factor de crecimiento epidérmico (EGF, Epidermal Growth Factor), el factor de crecimiento de transformación - $\beta$ (TGF- $\beta$,Transforming Growth Factor $\beta$ ) y el factor de crecimiento del endotelio vascular (VEGF, Vascular Endotelial Growth Factor). Diversos grupos han estudiado el papel de estos factores plaquetarios de crecimiento en la reparación de las heridas y el efecto perjudicial que conlleva su ausencia en los pacientes diabéticos con heridas de difícil manejo.

Los cambios temporales en la concentración de factores de crecimiento en el lecho vascular de las heridas, aunque no en su contraparte plasmática, indican la importancia de su papel en la reparación de las mismas. El uso sinérgico de diferentes factores de crecimiento ha mostrado mejorías significativas en la reparación de las heridas. El mecanismo por el cual las plaquetas inducen la proliferación vascular en las heridas es, en gran medida, incierto.

Los factores de crecimiento plaquetarios se almacenan en los gránulos $\alpha$ de las plaquetas. Estos de dividen en compartimentos pro y antiangiogénicos respectivamente, dependiendo de la naturaleza de los factores que almacenen. La degranulación selectiva y temporal de estimuladores y / o inhibidores de la angiogénesis, regulada por factores de crecimiento terciarios, culmina en la adecuada reparación de las heridas.
En modelos animales de plaquetas disfuncionales, la angiogénesis de novo se ve disminuida y la funcionalidad y madurez de los nuevos vasos sanguíneos es menor (1). Estudios in vitro muestran que las plaquetas también contribuyen a la reparación de las heridas estableciendo una red de soporte del proceso de granulación y epitelización. Además, se observan fragmentos plaquetarios en las extensiones de células endoteliales de los lechos vasculares de heridas de 5 a 9 días de evolución, que actúan como una férula sobre la cual se produce la neovascularización.

El trabajo en nuestros Departamentos de Cirugía Plástica y Medicina Vascular, se ha enfocado hacia la investigación del papel y de las propiedades de los factores antiangiogénicos, como el factor derivado del epitelio pigmentario (PEDF, Pigment Endothelium Derived Factor) en la fisiopatología de la retinopatía diabética y en la cicatrización defectuosa de heridas en pacientes diabéticos. También, en colaboración con otros centros hospitalarios, hemos logrado establecer el papel de los diferentes factores de crecimiento, como la Endogleína, en la fisiopatología de la preclampsia en pacientes diabéticas (2). Actualmente, nuestros Departamentos están trabajando en protocolos para el uso de preparaciones ricas plaquetas en la cicatrización defectuosa de heridas en pacientes diabéticos, centrándose en la diferencias en la concentración tisular de factores antiangiogénicos y en el posible mecanismo proangiogénico del uso de estas preparaciones ricas en plaquetas, a través de la inhibición de dichos factores antiangiogénicos.

\begin{tabular}{|l|l|l|l|l|l|}
\hline $\begin{array}{c}\text { Glicoproteínas } \\
\text { de Adhesión }\end{array}$ & Proteoglicanos & $\begin{array}{c}\text { Factores } \\
\text { Cofactores } \\
\text { Hemostáticos }\end{array}$ & $\begin{array}{c}\text { Mitógenos } \\
\text { Celulares }\end{array}$ & $\begin{array}{c}\text { Inhibidores } \\
\text { de Proteasas }\end{array}$ & \multicolumn{1}{|c|}{ Miscelánea } \\
\hline Fibronectina & PF4 & Fibrinógeno & PDGF & $\begin{array}{l}\alpha 2- \\
\text { Macroglobulina }\end{array}$ & IgG, IgA, IgM \\
\hline Vitronectina & BTG & $\begin{array}{l}\text { Factores V, } \\
\text { VII, XI, XII }\end{array}$ & TGF- $\beta$ & $\begin{array}{l}\alpha 2- \\
\text { Antitripsina }\end{array}$ & Albúmina \\
\hline Trombospondina & Serglicina & & ECGF & PDCI & GPIa \\
\hline vWF & HRGP & & EGF & $\alpha 2-$ Antiplasmina & \\
\hline & & & VEGF/VPF & PAI1 & \\
\hline & & & Interlucina - $\beta$ & $\alpha 2-$ PI & \\
\hline & & & & PIXI & \\
\hline & & & & PN2/APP & \\
\hline
\end{tabular}




\section{Encrucijadas en el uso clínico de las preparaciones plaquetarias}

El uso sinérgico de los diferentes factores de crecimiento constituye una dificultad práctica en el diseño de los protocolos de investigación y su uso clínico. Se ha citado el uso de gel de plaquetas autólogas, sin embargo, es difícil lograr predecir la proporción adecuada entre los diferentes factores involucrados en el proceso de reparación de las heridas, así como el momento adecuado para su administración.

Un abordaje alternativo sería el tratamiento con preparaciones de plasma rico en plaquetas (PRP). Estas pueden concentrar plaquetas y sus factores de crecimiento hasta 8 veces su concentración basal sin inducir la activación plaquetaria. El PRP se utiliza en la actualidad en combinación con injertos de hueso esponjoso para el tratamiento de defectos óseos en diferentes áreas de la Cirugía Plástica y Craneofacial, así como para el tratamiento de heridas recalcitrantes y del pie diabético (3).

El uso potencial de PRP autólogo elimina el riesgo de inmunogenicidad, de reacciones alérgicas y de transmisión de enfermedades infecciosas asociadas a riesgo transfusional. Sin embargo, estas preparaciones y el gel de plaquetas autólogas, han mostrado inconsistencias debidas a las características plaquetarias individuales de los pacientes que frecuentemente poseen comorbilidades debilitantes. Esto ha ocasionado resultados mixtos e inconclusos con respecto a la reparación de heridas (3).

El uso de plaquetas alogénicas sanas, permitiría la homogenización de las preparaciones de PRP; sin embargo, las reglas internacionales para el almacenaje de hemoderivados requieren que las plaquetas sean almacenadas a temperatura ambiente y desechadas 5 días después de su toma. Las plaquetas, retienen su actividad proliferativa hasta 21 días después de ser obtenidas y almacenadas a temperatura ambiente, pero esto acarrea un mayor riesgo de proliferación bacteriana y acumulación de citosinas pirógenas, limitando su uso en el campo de la reparación de heridas. Como resultado de estas regulaciones e impedimentos, se desperdician grandes volúmenes plaquetarios catalogados como caducos o no apropiados, lo que hace que los productos derivados de plaquetas sean costosos y posean una disponibilidad limitada, a pesar de que se obtienen cantidades mayores de las necesarias.

Existen varias estrategias diseñadas para prolongar la vida útil de las plaquetas, como el uso de PRP amortiguado con carbohidratos protectores como la sucrosa y la trehalosa.

La refrigeración $\left(4^{\circ} \mathrm{C}\right)$ y la criopreservación plaquetaria $\left(-80 \mathrm{a}-196^{\circ} \mathrm{C}\right)$ reducen el riesgo de colonización bacteriana, pero ocasionan activación plaquetaria irreversible. Se han propuesto métodos de estabilización modulando las vías intracelulares de la activación plaquetaria que se logran con bajas temperaturas, así como el uso de segundos mensajeros específicos como son el amiloride, la adenosina y el nitroprusiato de sodio (4).
Otro obstáculo con el que nos enfrentamos en el campo del tratamiento de las heridas recalcitrantes con preparaciones ricas en plaquetas es lo poco práctico que resulta el almacenaje de las plaquetas crioconservadas a $-196^{\circ} \mathrm{C}$, lo que ha dado lugar a la producción de preparaciones plaquetarias congeladas en seco (FD-PRP, Freeze Dried Platelet Rich Plasma).

A continuación revisaremos los protocolos recientemente diseñados para combatir los dilemas y encrucijadas actuales sobre el manejo de los diferentes productos derivados de las plaquetas, las más novedosas combinaciones y sus efectos positivos sobre la reparación de heridas, su almacenaje y manejo.

\section{Estabilización plaquetaria con Trehalosa}

Se ha observado que algunas plantas producen carbohidratos protectores en respuesta a temperaturas extremas y a la deshidratación celular sostenida. Estos carbohidratos conforman un enlace directo entre sus grupos hidroxilo y las superficies biomoleculares, desplazando las moléculas de agua, estabilizando el metabolismo celular y preservando la estructura y la función bioquímica de la célula. Estos compuestos retardan la difusión de radicales libres de oxigeno y nitrógeno, responsables de parte de la lesión celular. Al asociarse con dichos carbohidratos, las plaquetas pueden ser sometidas a congelación y deshidratación, preservando sus funciones endógenas de degranulación en respuesta al contacto con los estímulos in vivo (5).

La disponibilidad de un método de preservación plaquetaria podría disminuir los costos y aumentar la disponibilidad de estos hemoderivados, facilitando su almacenaje a temperatura ambiente (RT-PLT, Room Temperture Fresh Platelets), lo que minimiza la colonización bacteriana y viral de estas preparaciones, favoreciendo su manipulación.

En el año 2007, un grupo multihospitalario,finaciado por el Departamento de Estado y el Departamento de la Marina de los Estados Unidos de América, investigó los efectos del uso de preparaciones congeladas y deshidratas (FD, Freeze Dried) de plaquetas (FD-PLT) de edad menor a 5 días (FDPI, Stabilized Indated Freeze-Dried Platelets) o mayor a 5 días caducas (FDPO, Freíd-Dried Platelets Prepared From Outdated Platelets), comparando los efectos de estas preparaciones sobre la reparación de heridas y la biodisponibilidad de los factores de crecimiento plaquetarios utilizados como marcadores de la preservación de su función.

Las FDPI fueron preparadas con PRP de menos de 5 días desde su toma y las FDPO con PRP de 6 y 15 días. El PRP se mezcló con una solución compuesta por 85 $\mathrm{mM} / \mathrm{L}$ de citrato de sodio, $65 \mathrm{mM} / \mathrm{L}$ de acido cítrico y $111 \mathrm{mM} / \mathrm{L}$ de glucosa, balanceada a un $\mathrm{pH}$ de 6.6. El PRP se centrífugo durante 15 minutos para convertirlo en un tapón plaquetario. Después de eliminar el sobrenadante, este tapón se suspendió en una solución amortiguadora libre de cationes tipo Tyrode, con $9.5 \mathrm{mM} / \mathrm{L}$ de HEPES, 
$100 \mathrm{mM} / \mathrm{L}$ de $\mathrm{NaCl}, 4.8 \mathrm{mM} / \mathrm{L}$ de $\mathrm{KCl}, 12 \mathrm{mM} / \mathrm{L}$ de $\mathrm{NaHCO} 3$ y $50 \mathrm{mM} / \mathrm{L}$ de trehalosa balanceada a un $\mathrm{pH}$ de 6.8 y con un recuento plaquetario de $1.3 \times 10^{9} / \mathrm{ml}$ liofilizado con albúmina humana al 5\%. La suspensión se congeló durante una hora y se deshidrató a temperatura ambiente bajo presión negativa, alcanzando $\operatorname{los} 25^{\circ} \mathrm{C} \mathrm{du}-$ rante 12 horas y una humedad del 2 al 5\% (6).

Se eligieron 8 ratones genéticamente diabéticos, de 8 semanas de edad, Lep/r-db/db para cada grupo experimental, a los que se les produjo una herida de $1 \times 1 \mathrm{~cm}$ en piel y panículo carnoso. Los sujetos fueron asignados aleatoriamente a uno de los dos grupos y se les aplicó una dosis única de una de las dos preparaciones estudiadas sobre la herida. En el análisis in vitro, las FDPO de hasta 9 días mantuvieron su capacidad de promover el crecimiento, la proliferación celular y la angiogénesis, mostrando una actividad estimuladora similar a la de las preparaciones RTPLT y FDPI. Las FDPO también mostraron concentraciones de PDGF- $\beta \beta$ y de TGF- $\beta 1$ similares a las preparaciones de FDPI y RT-PLT. En el análisis in vivo, se observó que una sola aplicación de FDPI o FDPO causaba un cierre del $90 \%$ a los 17 días postrauma, en comparación con un $90 \%$ de cierre al día 21 en las heridas no tratadas. $\mathrm{La}$ aplicación de múltiples dosis de FDPO, proporcionó una reparación de la herida más eficiente que la aplicación de dosis únicas de estas preparaciones. También se observó un cierre de la herida del $90 \%$ en el día 12 con la administración de dosis múltiples, en comparación con un $90 \%$ de cierre en el día 17 en los grupos tratados con una dosis única y en el día 21 en el grupo no tratado.

En el día 9 del estudio se realizaron biopsias incisionales y análisis de las heridas no tratadas, así como de aquellas tratadas con dosis únicas y múltiples de FDPI y FDPO. En el grupo no tratado no hubo reepitelización, mientras que en los grupos tratados se observó una reacción reepitelizadora robusta. La administración de dosis múltiples de FDPO causó mayor área de reepitelización, así como hiperplasia en los bordes de la herida.

Este estudio demostró que las preparaciones congeladas y deshidratadas de plaquetas son efectivas en la estimulación in vivo e in vitro de la angiogénesis, la reepitelización y el cierre secundario de las heridas, manteniendo una concentración de factores de crecimiento plaquetarios adecuada hasta 5 días después de la fecha de caducidad establecida, lo que promete abrir la puerta a una reforma en las normas de tratamiento y amortiguación de las preparaciones plaquetarias y a un uso más eficiente de estos hemoderivados.

\section{Efectos del FD-PRP y comparación con FFP y PRP sonicado}

El grupo del Laboratorio de Ingeniería Tisular de la Universidad de Harvard, se ha enfocado en los últimos años a la comparación del FFP, FD-PRP, PRP sonicado (PRPs) y al uso de dermis alogénica en combinación con FD-PRP.
Las preparaciones de plaquetas que utiliza este grupo, se realizaron dividiendo un volumen plaquetario no caduco, en tres presentaciones de $1.2 \times 10^{6}$ plaquetas $/ \mu \mathrm{L}$. El PRPs se preparó ajustando la concentración plaquetaria de una de las presentaciones con Buffer B. Este amortiguador, se compone de $135 \mathrm{mM}$ de $\mathrm{NaCl}, 11.9 \mathrm{mM}$ de $\mathrm{NaHCO} 3,5.6 \mathrm{mM}$ de glucosa, 5mM de HEPES, $2.7 \mathrm{mM}$ de $\mathrm{KCl}, 2.0 \mathrm{mM}$ de $\mathrm{MgCl} 2$ y $0.42 \mathrm{mM}$ de $\mathrm{NaH} 2 \mathrm{PO} 4$ ajustado a un $\mathrm{pH}$ de 7.4. La preparación fue sonicada durante 10 segundos y congelada a $-80^{\circ} \mathrm{C}$ hasta su uso. El FDPRP se preparó ajustando la segunda presentación, con el Buffer B, y sometiendo la preparación a un congeladordeshidratador.

El FD-PRPp se preparó ajustando la tercera presentación con el Buffer B y una solución estabilizadora. Esta solución se compone de $250 \mu \mathrm{M}$ de amiloride, $100 \mu \mathrm{M}$ de adenosina, $50 \mu \mathrm{M}$ de nitroprusiato de sodio, $1 \%(\mathrm{v} / \mathrm{v})$ de sulfoxido dimetilo, $4 \%$ (v/v) de polivinilo de pirrolidona y $50 \mathrm{mM}$ de manitol. La preparación se congeló y deshidrató obteniendo FD-PRP preservado (FD-PRPp) (7). Las tres preparaciones se almacenaron en nitrógeno a temperatura de $-80^{\circ} \mathrm{C}$.

Se realizaron análisis in vitro de las concentraciones de PDGF-AB, TGF- $\beta$, VEGF y EGF en el PRPs y su sobrenadante libre de plaquetas, con el propósito de determinar la carga de factores de crecimiento, sus niveles plasmáticos, su distribución relativa entre los compartimentos intra y extracelular en la muestra sonicada y su redistribución después del procesamiento. También se determinó la habilidad plaquetaria para liberar factores de crecimiento, cuantificando la concentración plasmática de los mismos antes y después de la activación plaquetaria.

El modelo experimental fue un modelo de ratones diabéticos homocigotos para Lep/r-db/db, masculinos, de 8 semanas de edad, tipo C57BL/KsJ-Leprdb. La herida se realizó de la misma manera que en los experimentos descritos previamente. El PRPs se recalentó en agua tibia y las preparaciones de FD-PRP y FD-PRPp se rehidrataron hasta conseguir el volumen original predeshidratación; se separaron en dosis de $250 \mu \mathrm{L}$, cada una de las cuales se mezcló con $1 \mathrm{U} / \mathrm{ml}$ de Trombina justo antes de su aplicación, induciendo la agregación plaquetaria in situ. Las heridas se cubrieron con vendajes semioclusivos de poliuretano. Los animales fueron sacrificados a los 9 días de postoperatorio (POD); las heridas se fotografiaron y se resecaron para ser fijadas posteriormente en solución de formalina neutral al $10 \%$.

Se analizaron las fotografías de las heridas utilizando planimetría digital para cuantificar el área del tejido de granulación, el diámetro de la herida, el diámetro de epitelización y el diámetro de contracción de la herida con respectó a los sujetos no tratados. De estas observaciones se establecieron medidas criterio como la brecha epitelial (EP) y la brecha dérmica (DG) al POD 9 y se compararon con las del POD 0. La medida designada como cierre de herida es la suma de la contracción y re- 
epitelización y la brecha de herida $(\mathrm{BH})$, es una función del porcentaje que queda por cicatrizar.

Los cortes de las heridas resecadas fueron incubados con anticuerpos dirigidos contra el marcador nuclear de células panendoteliales MECA-32, el antígeno nuclear de proliferación celular (PCNA), la molécula de adhesión plaqueta-endotelio 1 (PECAM-1), y el antígeno CD34. Posteriormente, estos anticuerpos se utilizaron para demostrar los patrones de crecimiento angiogénico, de neovascularización, proliferación celular y densidad vascular.

\section{Efectos del FD-PRP y de los extractos de dermis mi- cronizada acelular sobre la reparación de heridas}

Hemos establecido que el plasma rico en plaquetas congelado y deshidratado (FD-PRP) estimula la angiogénesis temprana y la proliferación celular en modelos de cicatrización retardada y deficiente, tal y como la que muestran los modelos animales de ratones genéticamente diabéticos. Estas preparaciones ofrecen un método viable para extender la vida media de las preparaciones plaquetarias alogénicas a su uso en clínicas de tratamiento de heridas. En este punto nos encontramos con la dificultad para la aplicación de estas preapariciones plaquetarias, asociada con la activación de la trombina que estimula la liberación de factores plaquetarios, angiogénicos y antiangiogénicos en bolo.

Orgill y colaboradores realizaron un estudio en el modelo animal previamente descrito, evaluando los efectos biológicos e histológicos del uso de dermis micronizada alogénica en combinación con FD-PRP en comparación con el uso de estos productos por separado (8). Las preparaciones plaquetarias tipo FD-PRP se realizaron conforme a lo previamente descrito. La dermis micronizada alogénica se preparó a partir de muestras de dermis de ratones negros $C 57$, siguiendo los parámetros metodológicos tomados como base para realizar las preparaciones comerciales de dermis micronizada (9). Se utilizaron 187 $\mathrm{mg}$ de fragmentos de dermis de $200 \mu \mathrm{m}$ de diámetro y posteriormente se rehidrataron en $500 \mu \mathrm{L}$ de solución fisiológica (grupo 1) o de Buffer B (grupo 2). Las preparaciones de dermis obtenidas se congelaron y deshidrataron, obteniendo una proporción de matriz - solución hidratante de 2:1.

La dermis micronizada se incubó con PRP sonicado y el plasma residual se aisló posteriormente. La concentración de factores de crecimiento libre fue analizada y comparada con la concentración original de factores de crecimiento en la preparación de PRP, mostrando que la matriz de dermis micronizada retiene aproximadamente el 50\% del contenido inicial de factores de crecimiento del PRP, exceptuando al factor de crecimiento derivado de la insulina (IGF, Insulin Like Growth Factor). Las preparaciones de FD-PRP estabilizado mostraron una respuesta proliferativa y un mantenimiento de los factores de crecimiento en el lecho vascular de la herida similares a los del PRP, mientras que el uso de dermis micronizada sola no mostró respuesta proliferativa.
Por otro lado, la adición de PRP no estabilizado no causó un cambio significativo en la cinética de la reparación tisular, en comparación con la cicatrización espontánea de los controles a los 9 días de la lesión. Durante los primeros 9 días del estudio, se observó que el uso de dermis micronizada en combinación con FD-PRP estabilizado o FD-PRP no estabilizado, disminuyó los niveles de cierre de la herida; esto fue secundario a la disminución de la contracción de la herida en comparación con los controles. Los sujetos mostraron una disminución de 3 veces en el nivel de contracción de la herida cuando solo fueron tratados con dermis micronizada y una disminución de 1.5 veces cuando fueron tratados con dermis micronizada y FD-PRP estabilizado.

Se analizaron las heridas para establecer el área total de tejido nuevo, definido como tejido vascularizado y celularizado. El uso de dermis micronizada sola o con FDPRP aumentó el área de tejido nuevo en 2.8 y 3.8 veces respectivamente, en comparación con los sujetos control.

En el día 21 del estudio se observó que los tres grupos de tratamiento, FD-PRP, dermis micronizada y FD-PRP ( estabilizado o no ) más dermis micronizada, mostraron una mayor proporción de cierre de la herida y un resultado final favorable. Con respecto al área de tejido nuevo, se observó que todos los grupos tratados y los sujetos controles mostraron resultados similares. Sin embargo, la calidad de la herida cambio drásticamente.

Para estudiar la calidad del tejido cicatrizal neoformado y la distribución y densidad del colágeno nuevo, se tomaron biopsias de las heridas (en el día 21) y se tiñeron con tinción de Masson. Aquellas heridas tratadas con FD-PRP mostraron un patrón de inmadurez tisular con carencia de uniformidad y densidad de las fibras de colágeno. Por otro lado, las heridas tratadas con dermis micronizada, con o sin FD-PRP, mostraron fibras de colágeno menos densas, con mayor densidad celular, en comparación con los sujetos con FD-PRP y los sujetos controles.

También se analizaron las heridas para cuantificar el nivel de vascularizacion a través de la determinación del numero de vasos sanguíneo por campo de alto poder (hpf, high power field) a los 9 y a los 21 días. Durante estos dos periodos de tiempo, hubo tendencia hacia el aumento del numero de vasos sanguíneos por hpf en las heridas tratadas con FD-PRP solo o en combinación con dermis micronizada. En el día 9, el grupo tratado con FD-PRP mostró 12 vasos sanguíneos / hpf, mientras que el grupo tratado con FD-PRP y dermis micronizada mostró 11 vasos sanguíneos / hpf en comparación con 7 vasos sanguíneos / hpf en el grupo control no tratado. El numero de células en estado de proliferación presentes en el tejido neoformado, aumentó a niveles similares en los grupos tratados con FD-PRP y FD-PRP más dermis micronizada, alcanzando niveles de hasta un $22 \%$ en contraste con el $9 \%$ de los sujetos no tratados. Las heridas tratadas con FD-PRP mostraron una disminución sustan- 
cial del índice mitótico del tejido neoformado en el día 21 y solo aquellas heridas tratadas con dermis micronizada, sola o en combinación con FD-PRP, mostraron proliferación celular de hasta el $9.5 \%$.

El estudio concluye que a los 9 días de tratamiento, las heridas tratadas con FD-PRP, FD-PRP más dermis micronizada o dermis micronizada sola, muestran un aumento de la vascularización y de la proliferación celular en comparación con los controles no tratados. A los 21 días de tratamiento, aquellas heridas tratadas con FDPRP, FD-PRP más dermis micronizada o dermis micronizada sola muestran un aumento en su vascularización. Sin embargo, solo el uso de FD-PRP más dermis micronizada aumenta la proliferación celular en el lecho de la herida a los 21 días. Se propone que la deficiencia en la retención del factor de crecimiento IGF se debe a una disminución de la proteína de anclaje del IGF (IGF-BP IGF Binding Protein) o de los sitios de anclaje de sus cofactores. Esto se basa en la observación de que las IGF-BP forman enlaces con el colágeno tipo IV pero no con el tipo I, que es el que abunda en las preparaciones de dermis micronizada (10).

Es importante considerar que no todos los expansores tisulares basados en el colágeno tienen los efectos positivos que se observan con el uso de dermis micronizada. La dermis acelular micronizada se integra en el lecho de la herida y acelera y modula su reparación. Sin embargo, otros expansores tisulares como las cuentas de gelatina, no se integran en el tejido de la herida, sino que son rodeadas por una reacción tisular que conlleva la formación de una cápsula de tejido fibroso sin estimular la proliferación celular o la angiogénesis. La diferencia principal entre estas preparaciones a base de colágeno es que las estructuras bioquímicas terciaria y cuaternaria de las proteínas nativas de la matriz extracelular se preservan en la preparación de dermis micronizada acelular reteniendo sus efectos proangiogénicos, mientras que las cuentas de gelatina se conforman de colágena desnaturalizada en la que las estructuras terciaria y cuaternaria han sido distorsionadas por el propio proceso de creación. Además, el uso de dermis micronizada en conjunto con FD-PRP promueve una más rápida y densa neovascularización y proliferación celular durante los primeros 9 días, disminuyendo el área expuesta y el riesgo de infección que continúan siendo impedimentos importantes para el uso de rellenadotes tisulares y de otros productos avasculares. La presencia de un incremento de la proliferación celular con el uso de dermis micronizada, demuestra que las células nativas del lecho de la herida son capaces de migrar en el tejido alogénico y proliferar utilizándolo como matriz. Estos efectos tampoco se han visto con el uso de cuentas de gelatina, debido a la ausencia de puntos de unión efectivos. Los factores de crecimiento requieren su unión a las proteínas de la membrana extracelular para así ejercer sus propiedades angiogénicas, lo que se acentúa con el uso de dermis mi- cronizada en combinación con FD-PRP. También se ha visto que la combinación de grasa antóloga con PRP alarga la vida de los implantes de grasa usados para lipolifting de la región malar en procedimientos cosméticos (11).

Otro punto interesante es que el depósito excesivo de matriz extracelular en el lecho de la herida que se ve en los primeros 9 días desaparece posteriormente, previniendo la proliferación celular, el crecimiento de tejido o la hipertrofia tardías (día 21).

El uso de dermis micronizada en el estudio que analizamos, mostró una reducción en la contracción de la herida en el periodo temprano, a los 9 días, lo que puede ser útil en el tratamiento de heridas en áreas funcionales y en la preparación del lecho quirúrgico para algunos procedimientos quirúrgicos específicos.

Todos estos hallazgos dan pie para la realización de nuevos estudios enfocados a definir los efectos sinérgicos de los factores de crecimiento frente a su uso individual, así como su utilización en conjunto con diferentes concentraciones de preparaciones de dermis micronizada alogénica acelular. Serán pues necesarios estudios adicionales para determinar el volumen óptimo de dermis micronizada en el tratamiento de las heridas de difícil manejo y su efecto sobre los parámetros funcionales de la reparación de heridas tales como la fuerza tensil y las propiedades mecánicas del tejido neoformado.

\section{Condicionamiento plaquetario in vivo en un modelo tumoral}

Se ha visto que la concentración de factores angiogénicos tales como el VEGF, el factor básico de fibroblastos, el PDGF y el factor plaquetario 4, aumentan en las plaquetas de pacientes con neoplasias activas, pero no en su plasma (12). La integridad de la membrana plaquetaria y su sistema canalicular sirven como puertas para el control y regulación de la angiogénesis en las heridas de difícil reparación. En el estudio del proceso del establecimiento de las metástasis tumorales, se ha visto que las plaquetas son un factor importante para el desarrollo de nuevos vasos sanguíneos y propician por tanto el desarrollo de metástasis (13) a través de la secreción de sus productos y la adhesión al factor de Von Willebrand y a la fibronectina (14). El grupo de la Universidad de Harvard, realizó un estudio en el que se demuestra que las plaquetas condicionadas in vivo por la presencia de una neoplasia, muestran una mejor habilidad de degranulación de sus factores de crecimiento angiogénicos y mejoran la cicatrización de la heridas en comparación con preparaciones de plaquetas no condicionadas (15). En este estudio, ratones masculinos C57BL/6 sanos, fueron sometidos a un proceso de tumorogénesis a través de inyección de células de Lewis de carcinoma pulmonar. Tras valoración de la neoplasia y establecimiento del tamaño adecuado de la misma, los sujetos fueron exanguinados y se recolectó su volumen sanguíneo. Siguiendo 
los protocolos mencionados previamente, se realizaron preparaciones de PRP de los sujetos sometidos a tumorogénesis (TPRP) y del mismo numero de sujetos sanos (PRP). Se almacenó una muestra de cada preparación y el resto se utilizó para el manejo de dos grupos de sujetos con heridas en modelos similares a los descritos previamente. Se establecieron varios grupos: el grupo uno recibió PRP, mientras que el grupo dos recibió TPRP, ambos en la herida y en dosis única como hemos descrito previamente, mientras que el grupo tres no recibió tratamiento y actuó como grupo control.

El $33 \%$ de los sujetos de cada grupo fue seleccionado aleatoriamente y sacrificado a los 10 y 21 días respectivamente. Otro $33 \%$ fue seguido hasta el cierre total de las heridas, que fueron evaluadas según los parámetros mencionados previamente.

Las heridas que fueron tratadas con inyección única de TPRP presentaron un cierre superior al 50\% al cuarto día de la lesión, en comparación con un cierre del $20 \%$ en las heridas tratadas con PRP y un 5\% en los controles. Las heridas tratadas con TPRP mostraron un cierre del $90 \%$ a los 11 días de observación, con una reducción del tiempo de cierre del 90\% en 6 días y 10 días en comparación con las tratadas con PRP y los controles respectivamente.

Las heridas fueron examinadas histopatológica e immunohistoquímicamente con anticuerpos para PECAM-1, siguiendo los modelos descritos. Las heridas tratadas con TPRP mostraron una densidad de vasos sanguíneos neoformados de 61 vasos por hpf, mientras que las tratadas con PRP mostraron 44 vasos por hpf y los controles 15 vasos por hpf.

Para determinar el grado de angiogénesis activada por plaquetas, se evaluó el estado de fosforilación del receptor de VEGF (VEGFR-2/3) que es uno de los mayores reguladores de la angiogénesis. El tratamiento con TPRP y con PRP incrementó la fosforilación temprana de VEGFR-2/3 en los lechos vasculares de las heridas tratadas, en comparación con los controles.

La observación durante el día 21 mostró que las heridas tratadas con TPRP y PRP presentaban una disminución del número y densidad de vasos sanguíneos en comparación con la mostrada a los 10 días, mientras que los controles mostraron el efecto opuesto.

Las células estromales del tejido de granulación de las heridas tratadas con TPRP mostraron el nivel más alto de proliferación, con un $46 \%$ de las células en estados activos. Esto se estableció a través de tinción para el marcador de proliferación nuclear $\mathrm{Ki}$-67. Aquellas heridas tratadas con PRP mostraron un menor grado de proliferación celular que las tratadas con TPRP.

Durante la fase tardía de la cicatrización, el colágeno se deposita y organiza para formar una cicatriz estable. El tratamiento de las heridas con TPRP y PRP incrementó la formación y el deposito de colágeno en comparación con los controles. Sin embargo, el patrón de depósito del colágeno difirió entre estos dos tipos de tratamiento. El uso de TPRP ocasionó no solo un aumento en el deposito de colágeno, sino una secreción de fibras más gruesas alternando con áreas de alta celularidad. En contraste, el uso de PRP ocasionó el depósito de un colágeno con fibras delgadas y relativamente celular, característico de las cicatrices en esta etapa de su formación.

Los autores mostraron que la congelación de PRP y de TPRP ocasiona la pérdida de sus propiedades angiogénicas pero no de las proliferativas. Esta observación se atribuye a la activación irreversible de las plaquetas al ser congeladas y recalentadas.

Este grupo de estudio concluye que el condicionamiento tumoral plaquetario estimula la angiogénesis en el $10^{\circ}$ día, lo que se sigue de una pobre regulación a la baja del crecimiento vascular en las fases tardías de la cicatrización. Esto concuerda con el hecho de que, en las heridas benignas, el aumento de la trombina y el contenido de colágeno causen activación del receptor activado por Proteasa 4 que estimula la liberación de mediadores antiangiogénicos de las células estromales y las plaquetas (15). En contraste, la proliferación y la expresión continua de factores proangiogénicos en las células tumorales previene la regulación a la baja de la angiogénesis y de la proliferación celular en los tejidos malignos. La arquitectura distintiva de la matriz extracelular de los lechos vasculares de las heridas tratadas con TPRP sugiere que existe una estimulación directa ejercida por las plaquetas sobre la proliferación estromal. No queda claro si la activación estromal es secundaria o si es la causa de la angiogénesis.

El estudio también mostró que aunque no existe una diferencia cuantitativa en la concentración de factores de crecimiento como el VEGF, el factor de crecimiento básico de fibroblastos o el PDGF, las heridas tratadas con TPRP mostraron una mayor angiogénesis, lo que sugiere una presentación más efectiva de estos factores de crecimiento por las plaquetas condicionadas. Esto se apoya al observar que la angiogénesis fue inducida solo por el uso de plaquetas intactas, pero no por las preparaciones congeladas ni por la exposición aislada a factores del crecimiento. Todos estos hallazgos sugieren que el estudio tumoral y su interacción con las vías angiogénicas puede llevar al desarrollo de métodos clínicos eficaces para el tratamiento de las heridas de difícil tratamiento.

\section{Conclusiones}

El uso de preparaciones plaquetarias estimula la angiogénesis, la neovascularización y la reparación de las heridas. Las plaquetas actúan como un molde sobre el cual se depositan las células endoteliales de los nuevos vasos sanguíneos. Actúan también como secretagogos bioquímicos de factores de crecimiento pro y antiangiogénicos que orquestan la reacción tisular a la inflamación y el reclutamiento de células progenitoras del tejido conectivo.

El uso y manejo de las plaquetas presenta dificultades debido a su proceso de preservación y la potencial transmisión de enfermedades infecciosas. El almacenaje 
de las preparaciones plaquetarias en criopreservación, supone un problema que limita su uso a hospitales con la infraestructura adecuada para llevar a cabo dicho proceso. Frente a esta encrucijada se han desarrollado diferentes estrategias como la congelación y deshidratación de las preparaciones plaquetarias y el uso de plaquetas catalogadas como caducas, por su edad, siguiendo las normas y directrices internacionales de transfusión de hemoderivados. Está probado que el uso de plasma rico en plaquetas (PRP), así como de sus diferentes presentaciones congeladas, congeladas y deshidratadas, sonicadas y estabilizadas, confiere importantes beneficios al tratamiento de las heridas recalcitrantes.

Por último, proponemos el seguimiento de los estudios actuales que relacionan la tumorogénesis y el proceso metastático y sus efectos sobre las plaquetas in vivo e in vitro, con un condicionamiento y un efecto favorable sobre la cicatrización de heridas en sujetos que no poseen dichos tumores. Es importante que como cirujanos plásticos nos mantengamos en constante actualización sobre estos temas que se encuentran actualmente en evolución y revolución.

\section{Dirección del autor}

Dr. David A. Mateo de Acosta

Advocate Illinois Masonic Medical Center

836 W Wellington Avenue Room 4807,

Chicago IL 60657. U.S.A.

e-mail: drdavidmateodea@yahoo.com.mx

\section{Bibliografía}

1. Eppley BL, Woodell JE, Higgins J.: "Platelet quantification and growth factor analysis from platelet-rich plasma: implications for wound healing". Plast Reconstr Surg 2004; 114: 1502 .

2. Yu Y., Jenkins A., Nankervis A., Hanssen K., Scholz H., Henriksen T., Clausen $\mathbf{S}$ y cols.: "Anti-angiogenic factors and pre-eclampsia in type 1 diabetic women". Diabetologia 2009;52:160.

3. Montón Echeverria J., Pérez Redondo S., Gómez Bajo G.: "Experiencia clínica en el empleo de factores de cre- cimiento autólogos, obtenidos de plasma rico en plaquetas". Cir. plást.iberolatinoam. 2007; 33 (3):155.

4. Crowe JH, Tablin F, Wolkers WF, et al.: "Stabilization of membranes in human platelets freeze-dried with trehalose". Chem Phys Lipids 2003;122:41.

5. Read MS, Reddick RL, Bode AP, et al.: "Preservation of hemostatic and structural properties of rehydrated lyophilized platelets: potential for long-termstorage of dried platelets for transfusion". Proc Natl Acad Sci U S A 1995;92:397.

6. Wolkers WF, Tablin F, Crowe JH.: "From anhydrobiosis to freeze-drying of eukaryotic cells." Comp Biochem Physiol A Mol Integr Physiol 2002;131:535.

7. Sum, R, Hager S, Pietramaggiori G., Orgill DP., Dee J., Rudolph A., Orser C., Fitzpatrick GM, Ho D.: "Wound-healing properties of trehalose-stabilized freeze-dried outdated platelets". Transfusion 2007;47:672.

8. Pietramaggiori G, Scherer S, Mathews J, Alperovich M, Yang H, Neuwalder J, Arch M, Czeczuga, Orgill D.: "Healing modulation induced by freeze-dried platelet-rich plasma and micronized allogenic dermis in a diabetic wound model". Wound Rep Reg, 2008, 16: 218.

9. Maloney BP, Murphy BA, Cole HP 3rd.: "Cymetra". Facial Plast Surg 2004; 20: 129.

10. Martin JL, Jambazov S.: "Insulin-like growth factor binding protein-3 in extracellular matrix stimulates adhesion of breast epithelial cells and activation of p44/42 mitogenactivated protein kinase". Endocrinology 2006; 147:4400.

11. Serra Renom, J.M., Muñoz del Olmo, J.L. and Gonzalo Caballero, C.: "Uso de factores de crecimiento plaquetar unidos a injertos de grasa para lipofiling facial en ritidectomía”. Cir. plást. iberolatinam.2006,32 (3): 191.

12. Cervi D, Yip TT, Bhattacharya N, Podust VN, Peterson J, Abou-Slaybi A, Naumov GN, Bender E, Almog N, Italiano JE Jr., Folkman J, Klement GL: "Plateletassociated PF-4 as a biomarker of early tumor growth". Blood 2008, 111:1201.

13. Trikha M, Nakada MT: "Platelets and cancer: implications for antiangiogénica therapy". Semin Thromb Hemost 2002, 28:39.

14. Karpatkin S, Pearlstein E, Ambrogio C, Coller BS: "Role of adhesive proteins in platelet tumor interaction in vitro and metastasis formation in vivo". J Clin Invest 1988, 81:1012.

15. Pietramaggiori G, Scherer S, Cervi D, Klement G, Orgill D.: "Tumors Stimulate Platelet Delivery of Angiogenic Factors in Vivo An Unexpected Benefit". AJP 2008, 173:6 1609. 